\title{
Diseño e implementación de una máquina recicladora de botellas plásticas por corte, controlada automáticamente
}

\section{(Design and implementation of an automatically controlled plastic bottles cutting recycling machine)}

\author{
Luis Hidalgo Aguilera ${ }^{1}$, Jimmy Imbaquingo ${ }^{1}$, Daniel Mideros ${ }^{1}$
}

\begin{abstract}
Resumen:
Se ha diseñado una máquina automática para la obtención de hilos y cintas mediante un proceso de corte rotacional continuo. Esta máquina procesa botellas plásticas usadas como envases para almacenamiento y transporte de bebidas gaseosas y no gaseosas con capacidad de tres litros. Es un diseño mecatrónico, compuesto por sencillos sistemas mecánicos/neumáticos, eléctricos y de control, que permite procesar hasta cincuenta botellas por hora. Se puede obtener una variedad de productos comprendidos entre aproximadamente $46.5 \mathrm{~m}$ de longitud de hilo de 1.6 $\mathrm{mm}$ de espesor (ancho) y $11.3 \mathrm{~m}$ de longitud de cinta de $6.7 \mathrm{~mm}$ de ancho por botella. Estos productos son materia prima económica obtenida de material de desecho como son las botellas plásticas, elementos altamente contaminantes del ambiente por el pequeño porcentaje que es reciclado especialmente en Ecuador. Esta materia prima puede tener aplicaciones en algunos procesos de manufactura como son: la fabricación de escobas, cuerdas, canastos, fundas, elementos sujetadores entre otros.
\end{abstract}

Palabras clave: contaminante; envases; máquina; plástico; reciclaje.

\begin{abstract}
:
An automatic machine has been designed to obtain threads and ribbons through a continuous rotational cutting process. This machine processes plastic bottles used as containers for storage and transportation of soft drinks and non-carbonated drinks with a capacity of three liters. It is a mechatronic design, composed of simple mechanical / pneumatic, electrical and control systems, which allows to process up to fifty bottles per hour. A variety of products can be obtained; from threads with approximately 46.5 $\mathrm{m}$ in length and $1.6 \mathrm{~mm}$ thick (width) to ribbons with $11.3 \mathrm{~m}$ in length and $6.7 \mathrm{~mm}$ in width per bottle can be obtained. These products are economic raw material obtained from waste material such as plastic bottles, which are highly polluting elements of the environment due to the small percentage that is recycled especially in Ecuador. This raw material can have applications in some manufacturing processes such as the manufacture of brooms, ropes, baskets, bags, fasteners among others.
\end{abstract}

Keywords: contaminant; containers; machine; plastic; recycling.

\footnotetext{
1 Universidad Tecnológica Equinoccial, Quito - Ecuador (\{lahidalgo, icjd92912, dmideros\}
} @ute.edu.ec). 


\section{Introducción}

"La mitad del plástico que producimos se convierte en residuo en cuatro o cinco años" (Geyer, Jambeck, \& Lavender Law, 2017). Se calcula que desde que se inventó el plástico hasta el año 2015 se han producido unos 8300 millones de toneladas métricas que correspondería a unos $1100 \mathrm{Kg}$ por habitante en el mundo. De esta cantidad de plástico que se ha producido en la historia de la humanidad, todavía está en uso aproximadamente un $30 \%$ es decir unas 2490 toneladas métricas, el $70 \%$ restante se ha convertido en residuos. Del total de estos residuos, el $79 \%$ ha sido arrojado en cualquier lugar del planeta contaminando el ambiente o en algún vertedero, el $12 \%$ ha sido incinerado y tan solo el $9 \%$ que significa aproximadamente unas 540 toneladas ha sido reciclado. Si continúa la tendencia actual de fabricación y gestión de plásticos, para el año 2050 habrá 13000 millones de toneladas de plásticos arrojadas en los vertederos o en el ambiente. Respecto a la incineración como medio de "eliminación" del plástico, no es nada aconsejable, debido a la emisión de gases y partículas perjudiciales para la salud y el ambiente. (Geyer, Jambeck, \& Lavender Law, 2017).

En el Ecuador, al igual que en el mundo entero existe una gran preocupación por la eliminación de los desechos plásticos "sólidos" tal como las botellas utilizadas como recipientes contenedores principalmente de líquidos, las mismas que no son biodegradables razón por la cual resulta demasiado costosa tanto su presencia en el ambiente como sus intentos de eliminación.

Según el INEC (Instituto Ecuatoriano de Estadísticas y Censos), en el año 2014, el $11.78 \%$ de los residuos sólidos recolectados a nivel nacional por los Municipios, corresponde a los plásticos; es el porcentaje más alto después de los residuos orgánicos que constituyen el $61,12 \%$ (INEC, 2015). De acuerdo con la misma fuente, en los últimos seis años, a nivel nacional los hogares muestran una tendencia a clasificar en mayor proporción el plástico, seguido por residuos orgánicos, papel / cartón y finalmente el vidrio, lo que ha dado como resultado que para el año 2015, esta proporción de plástico alcance el 32.7\% (INEC, 2015).

Existen sistemas de reciclaje de desechos plásticos "solidos", los cuales en su gran mayoría son procesos de compactación y trituración, (García Villalba, Ponce Corral, Martínez López, \& León Ordaz, 2016) cuyos productos son utilizados como complemento en procesos energéticos o de infraestructura (Pereira de Oliveira \& Castro-Gomes, 2011), mas sin embargo, es muy poco lo que se ha trabajado en proyectos que involucren directamente la reutilización de las botellas plásticas, como por ejemplo la obtención de fibras en forma de hilos a partir de las botellas (Damián Eneque, 2016); permitiendo así la integración de esta actividad a un nuevo ciclo económico, generando la reutilización como materia prima para la elaboración de nuevos productos.

Por lo mencionado, el objetivo principal de este proyecto es diseñar e implementar una máquina automática para la obtención de hilos y cintas mediante un proceso de corte rotacional continuo a partir de botellas plásticas usadas como envases para almacenamiento y transporte de bebidas gaseosas y no gaseosas con capacidad de tres litros.

Los beneficios que se lograrán mediante la implementación de este dispositivo de reciclaje serán: la disminución considerable de contaminación que estos producen y una rentabilidad a todos los actores en el proceso, ya que las botellas plásticas desechables serán convertidas en nueva materia prima que puede ser utilizada como fibra para escobas, hilos para uso agrícola y confección de artesanías entre otros.

\subsection{Etapas del reciclaje}

Recolección: proceso de recogida de residuos plásticos separándolos de los orgánicos e inorgánicos. Por lo general este proceso lo inician personas recolectoras de 
residuos en los basureros o lugares de acopio de basura, actividad a través de la cual han encontrado un medio de sustento.

Centro de Reciclaje: lugar en donde se reciben los residuos plásticos, para su posterior tratamiento.

Clasificación: proceso por el cual se agrupan los residuos plásticos de acuerdo con el tipo y color de plástico. En esta esta, se valoran los residuos que pueden ser reciclados. La etapa final consiste en el reciclaje.

Reciclaje: proceso por el cual se transforman física, química o biológicamente los desechos plásticos con el objetivo de obtener nueva materia prima con la que se elaborarán los mismos o diferentes productos. (Nadales Díaz, 2017)

Hay que tener en cuenta que si bien el reciclaje mecánico tiene un mejor perfil ambiental que el químico, las fibras químicamente recicladas tienen una gama más amplia de aplicaciones que las fibras recicladas mecánicamente. (Shen, Worrell, \& Patel, 2010)

\subsection{Tipos de máquinas recicladoras}

Existen una gran variedad de máquinas que son utilizadas en el reciclaje de botellas plásticas, las mismas que pueden ser agrupadas de la siguiente manera:

1.2.1 Compactadoras: permiten reducir considerablemente el volumen de los residuos reciclados mediante la aplicación de una fuerza de compresión sobre los residuos.

1.2.2 Trituradoras: permiten producir materiales de menor tamaño a partir de los materiales originales, esto se logra mediante la molienda de los materiales desechados.

1.2.3 Granuladoras: se encarga de reducir el tamaño del material obtenido de la trituradora para convertirlo en pequeños pedazos llamados gránulos o grageas.

1.2.4 Máquinas cortadoras: permiten extraer hilos de diferentes tamaños de las botellas plásticas recicladas mediante corte continuo. Pueden ser accionadas manualmente o por medio de un motor y de acuerdo con la posición de la botella pueden ser horizontales o verticales.

- Máquina cortadora vertical: las botellas son colocadas en forma vertical en la cortadora, por lo general estas deben tener la base ya previamente cortada para su funcionamiento, en este tipo de máquina se pueden procesar hasta 5 botellas al mismo tiempo, pero es necesario la intervención del operador para colocarlas.

- Máquina cortadora horizontal: las botellas son colocadas en forma horizontal, por lo general no es necesario la extracción de la base para su proceso, se puede procesar una sola botella y es necesaria la intervención del operador para sujetar la botella en la máquina.

Conviene aclarar que las máquinas cortadoras de botellas con el propósito de hacer hilos que se pueden encontrar localmente en el Ecuador son demasiado básicas y nada automáticas, por lo cual el proyecto se convierte en algo inédito que: contribuirá a reducir la contaminación ambiental, a generar fuentes de ingreso y a la elaboración de materia prima que podrá ser utilizada de manera directa o en la producción de otros elementos plásticos. 


\section{Metodología}

Para el diseño de la máquina se siguió la metodología del modelo en $\mathrm{V}$ o de cuatro niveles, que es una representación gráfica de las relaciones temporales entre las distintas fases del ciclo de desarrollo de un proyecto (Inteco, 2009). Lo que significa que para cada fase del desarrollo existe una fase correspondiente o paralela de verificación o validación, es decir que para cada fase del desarrollo debe existir un resultado verificable (Flores, 2009).

\subsection{Diseño geométrico / funcional de la máquina}

- Requerimientos: como todo sistema mecatrónico, se determinó en la máquina un sistema mecánico/neumático, uno eléctrico/electrónico y uno de programación debidamente integrados de tal manera que se garantice la consecución de los objetivos planteados para el proyecto. Además se definieron rangos de valores para las variables que intervienen en el proceso como son: capacidad de producción, capacidad de almacenamiento, número y tipo de funciones, tipo de energía, interface hombre máquina. Para la materia prima se establecieron las dimensiones y condiciones de las botellas a procesar.

- Limitaciones y restricciones: se identificaron los procesos en los cuales se necesita la intervención del operador y se determinó que el tipo de materia prima a utilizar influye en el diseño de la máquina.

- Diseño general del sistema: se estableció de forma general la estructura del sistema con todos los elementos que debían conformar la máquina para su funcionamiento y la manera de cómo estos elementos debían interactuar entre sí.

\subsection{Diseño específico de la máquina}

- Para el diseño y/o selección de los elementos que conforman el sistema mecánico, se consideraron tanto los esfuerzos como las deformaciones máximas a las cuales estarían sometidos según el tipo de carga que se ejerzan sobre ellos, cuando la máquina opera a plena carga, cuidando que en ningún caso sobrepasen los permisibles establecidos para el material o el elemento mismo, además se tomó en cuenta que las dimensiones y características estén de acuerdo con las establecidas en los requerimientos (Budynas \& Nisbett, 2008). Este sistema básicamente consta de: estructura, tolva de alimentación, ejes, tornillos sin fin, poleas, bandas, caja reductora y carrete de producto.

Tanto para el modelado mecánico y especialmente para la validación del diseño se hizo uso del software SolidWorks Simulation que determina el comportamiento de los desplazamientos de cada uno de los nodos, la deformación unitaria, las tensiones para cada uno de los puntos y del factor de seguridad, el mismo que como mínimo debe tener un valor de 2.5 de acuerdo con las recomendaciones de diseño (Gómez González, 2010).

- De acuerdo con las funciones que son: controlar el sistema de distribución, controlar el sistema de elevación, controlar el sistema sujetador y controlar el sistema de corte y con las cargas que deben soportar los elementos neumáticos que se muestran en la Tabla 1 , al realizar estas funciones se diseñó el circuito correspondiente en el software Festo FluidSim (Valentín Labarta, 2012) de tal manera de poder simularlo y validarlo, luego de lo cual se seleccionaron los siguientes elementos: compresor, cilindros, electroválvulas, cañerías y accesorios complementarios (Serrano, 2009). 
Tabla 1. Datos de los cilindros neumáticos.

\begin{tabular}{|l|c|c|c|}
\hline Sistema & $\begin{array}{c}\text { Carrera } \\
(\mathbf{m m})\end{array}$ & $\begin{array}{c}\text { Fuerza } \\
\text { necesaria (N) }\end{array}$ & $\begin{array}{c}\text { Fuerza cilindro } \\
\text { seleccionado } \\
(\mathbf{N})\end{array}$ \\
\hline Distribución & 125 & 23.94 & 120 \\
\hline Elevación & 200 & 47.91 & 120 \\
\hline Punzón & 50 & 47.91 & 120 \\
\hline Corte & 50 & 217.87 & 228 \\
\hline
\end{tabular}

- De acuerdo con las funciones que son: actuadores rotacionales como los motores, actuadores lineales como los cilindros neumáticos, sensores como los finales de carrera y de presencia; e indicadores y mando y las cargas de trabajo que deben soportar estos y otros elementos eléctricos / electrónicos complementarios se diseñó el circuito correspondiente a base del cual se seleccionaron los siguientes elementos: motores eléctricos, electroválvulas, contactores, disyuntor, relés, sensores, tarjeta de control, HMI, cables y accesorios complementarios (Bolton, 2016).

Para el diseño del circuito de control se utilizó el software PROTEUS 8.5 SP (Tojeiro Calaza, 2014), esta herramienta permitió diseñar el circuito electrónico, el circuito impreso, desarrollar el software y realizar pruebas de funcionamiento.

La PCB, sensores y actuadores son alimentados con una fuente conmutada que entrega 5 VDC - 5 A, 12 VDC - 1.5 A, para la PCB y los sensores se utiliza 5VDC, los motores de corriente continua son alimentados por una fuente adicional de 12 VDC - 22 A, las fuentes son conectadas directamente a la red eléctrica 220VAC.

- Se realizó la lógica de control de la máquina y el código para controlar la tarjeta PCB. Se hizo uso de los softwares: Bascon AVR para programar el microcontrolador en lenguaje Basic y Nextion Editor para desarrollar el interfaz de control y visualización entre el humano y la máquina (Siemens, 2015).

\subsection{Pruebas de validación del funcionamiento de la máquina}

Con el propósito de confirmar que la máquina cumplía con los requerimientos y el objetivo principal del proyecto planteado en la introducción del documento, es decir de validar su funcionamiento, se realizaron las siguientes pruebas:

- El funcionamiento en vacío de cada uno de los sistemas que la componen.

- El funcionamiento del sistema de control de acuerdo con la lógica de control establecida para la misma.

- El funcionamiento de la máquina tanto en modo semiautomático como en automático.

- Tiempos de corte.

- Grosor (ancho) del hilo (cinta) obtenido.

- Cantidad de fibra obtenida por botella.

- Calidad de corte.

\section{Resultados y Discusión}

\subsection{Características generales de la Máquina}

En la Tabla 2, se muestran los valores o el comportamiento de las características generales de la máquina. 
Tabla 2. Características generales de la máquina.

\begin{tabular}{|l|l|}
\hline \multicolumn{1}{|c|}{ Características } & \multicolumn{1}{c|}{ Valores / Comportamiento } \\
\hline Peso & $120 \mathrm{Kg}$ aproximadamente \\
\hline Dimensiones & $1800 \times 1250 \times 800 \mathrm{~mm}$ \\
\hline Ruedas para su movilidad & 4 \\
\hline Movilidad & Fácil en el área de trabajo \\
\hline
\end{tabular}

\subsection{Características Técnicas}

En la Tabla 3, se muestran los valores o el comportamiento de las características técnicas de la máquina.

Tabla 3. Características técnicas de la máquina.

\begin{tabular}{|l|l|}
\hline \multicolumn{1}{|c|}{ Características } & \multicolumn{1}{c|}{ Valores / Comportamiento } \\
\hline Funciones & Corte automático y semiautomático \\
\hline Capacidad de proceso & 30 botellas de 3 litros por hora \\
\hline Suministro eléctrico & AC $220 \mathrm{~V}$, bifásico \\
\hline Potencia & $1.5 \mathrm{~kW}$ \\
\hline Requerimientos & Aire comprimido a 40 psi de presión \\
\hline Control & Mediante HMI \\
\hline Opciones & Monitoreo de productividad \\
\hline Riesgos específicos & Atascamiento y corte \\
\hline
\end{tabular}

\subsection{Componentes seleccionados}

a) Un sistema de almacenamiento y distribución

b) Un sistema de elevación

c) Un sistema sujetador

d) Un sistema de transporte

e) Un sistema de corte

f) Un sistema de enrollamiento

La Figura 1 muestra una vista explosionada de los componentes de cada uno de los sistemas de la máquina recicladora por corte.

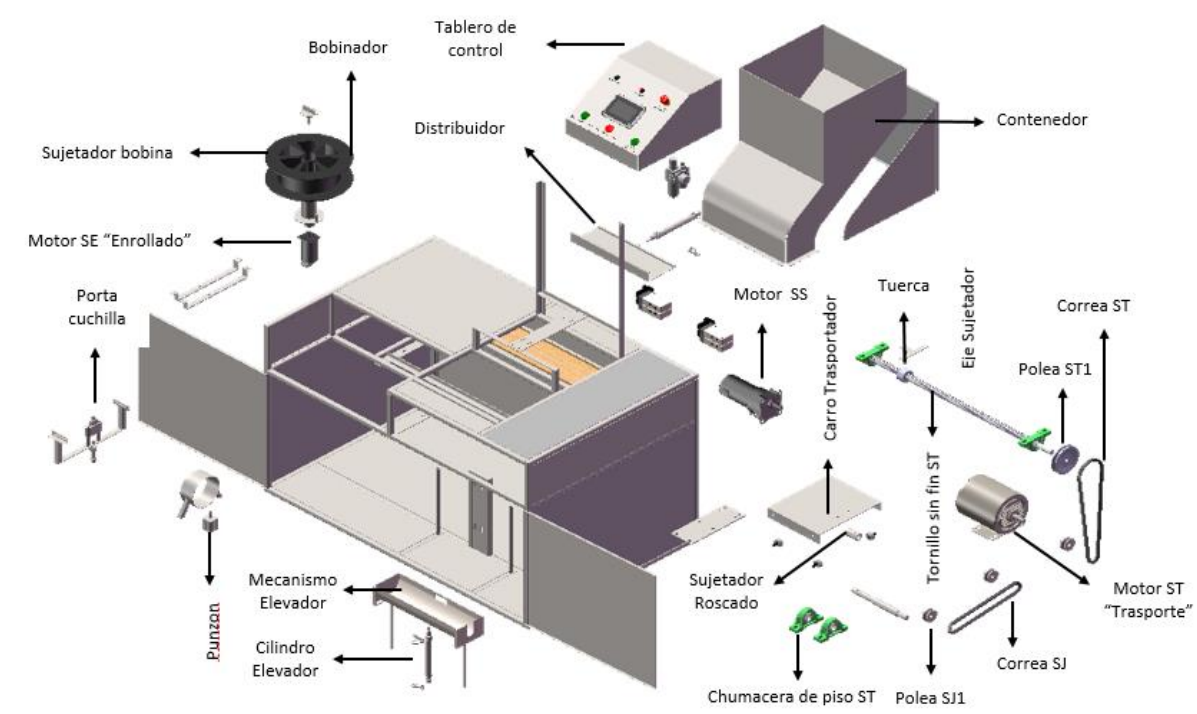

Figura 1. Máquina recicladora por corte. 
La Tabla 4, muestra, el material o marca y la cantidad de los elementos que componen los sistemas de almacenamiento y distribución, de elevación, sujetador, de transporte, de corte, de enrollamiento, y neumático de la máquina

Tabla 4. Componentes de la máquina recicladora.

\begin{tabular}{|c|c|c|c|}
\hline \multicolumn{4}{|c|}{ Lista de componentes de la máquina recicladora } \\
\hline Sistema & Elementos & Cantidad & Material \\
\hline \multirow{3}{*}{$\begin{array}{c}\text { Sistema de } \\
\text { almacenamiento } \\
\text { y distribución }\end{array}$} & Contenedor & 1 & ASTM 570 \\
\hline & Mecanismo distribuidor & 1 & ASTM A36 \\
\hline & Estructura tubo cuadrado de $3 / 4 "$ & 1 & ASTM A36 \\
\hline \multirow{2}{*}{$\begin{array}{l}\text { Sistema de } \\
\text { elevación }\end{array}$} & Elevador & 1 & ASTM A36 \\
\hline & Base para elevador & 1 & ASTM A36 \\
\hline \multirow{7}{*}{ Sistema sujetador } & Poleas de 2.5 in & 2 & ALUMINIO \\
\hline & Banda Tipo A36 & 1 & DAYCO \\
\hline & Eje sujetador & 1 & AISI 1018 \\
\hline & Base roscada & 1 & AISI 1018 \\
\hline & Motor DC de $1 / 4 \mathrm{Hp}, 330 \mathrm{Rpm}$ & 1 & KING \\
\hline & Chumaceras de $3 / 4 "$ & 2 & NTN \\
\hline & Base & 1 & ASTM A36 \\
\hline \multirow{9}{*}{$\begin{array}{l}\text { Sistema de } \\
\text { transporte }\end{array}$} & Polea de 4.5" & 2 & ALUMINIO \\
\hline & Banda Tipo A40 & 1 & DAYCO \\
\hline & Motor Trifásico de $1 / 4 \mathrm{Hp}$ & 1 & WEG \\
\hline & Variador de Frecuencia de $0.37 \mathrm{KW}$ & 1 & OMRON \\
\hline & Chumaceras $3 / 4$ & 2 & NTN \\
\hline & Tornillo sin fin & 1 & AISI 1018 \\
\hline & Base para carro & 1 & ASTM A36 \\
\hline & Rodamientos 6200 & 4 & NTN \\
\hline & Ejes para rodamiento & 4 & AISI 1018 \\
\hline \multirow{4}{*}{ Sistema de corte } & Portacuchillas & 1 & ALUMINIO \\
\hline & Chuchilla & 1 & INOX \\
\hline & Guía de corte & 1 & ASTM A36 \\
\hline & Eje guiador & 1 & ASTM A36 \\
\hline \multirow{4}{*}{$\begin{array}{l}\text { Sistema de } \\
\text { enrollamiento }\end{array}$} & Soporte para caja reductora & 1 & ASTM A36 \\
\hline & Soporte para bobina & 1 & ASTM A36 \\
\hline & Bobina & 1 & PLASTICO \\
\hline & Motor DC 1/4 Hp, 20 Libras, 220 Rpm & 1 & KING \\
\hline \multirow{10}{*}{$\begin{array}{c}\text { Sistema } \\
\text { neumático }\end{array}$} & Unidad de mantenimiento $1 / 8$ " a 8 PSI & 1 & AIRTAC \\
\hline & Reguladores de flujo & 8 & FESTO \\
\hline & Cilindro neumático (16mmx125mm) & 1 & AIRTAC \\
\hline & Cilindro neumático $(16 \mathrm{~mm} \times 200 \mathrm{~mm})$ & 1 & AIRTAC \\
\hline & Cilindro neumático (16mmx50mm) & 1 & AIRTAC \\
\hline & Cilindro neumático (22mmx50mm) & 1 & MAIDMAN \\
\hline & Compresor 1/3 HP, $100 \mathrm{PSI}$ & 1 & PORTER \\
\hline & Cañería, diámetro $4 \mathrm{~mm}$ & $20 \mathrm{~m}$ & FESTO \\
\hline & Electroválvulas 5/2 monoestable $110 \mathrm{v}$ & 4 & AIRTAC \\
\hline & Accesorios y acoples & varios & AIRTAC \\
\hline
\end{tabular}

Tanto la Figura 1 como las Tabla 4 y 5 muestran todos los componentes de la máquina recicladora de botellas plásticas por corte que se obtuvo como resultado final 
con sus diferentes sistemas, integrados para su funcionamiento de acuerdo con los objetivos planteados por medio de un sistema eléctrico/ electrónico, un sistema neumático y una lógica de programación.

En la Tabla 5, se muestran los componentes de los sistemas eléctrico y de control de la máquina.

Tabla 5. Componentes del sistema eléctrico y de control de la máquina recicladora.

\begin{tabular}{|l|l|c|c|}
\hline \multicolumn{3}{|c|}{ Lista de componentes de la máquina recicladora } \\
\hline Sistema & \multicolumn{1}{|c|}{ Elementos } & Cantidad & Material \\
\hline \multirow{5}{*}{ Sistema eléctrico } & 2 & SCHNEIDER \\
\cline { 2 - 4 } y de control & Breaker 2P & 2 & SASSIN \\
\cline { 2 - 4 } & Portafusibles & 1 & CHINT \\
\cline { 2 - 4 } & Contactor & 3 & CAMSCO \\
\cline { 2 - 4 } & Relé auxiliares 8 pines 110 VAC & 1 & \\
\cline { 2 - 4 } & Fuente conmutada & 2 & CAMSCO \\
\cline { 2 - 4 } & Luz piloto verde 22 mm & 1 & CAMSCO \\
\cline { 2 - 4 } & Luz piloto rojo 22 mm & 1 & CAMSCO \\
\cline { 2 - 4 } & Pulsadores verde & 1 & CAMSCO \\
\cline { 2 - 4 } & Pulsadores rojo & 1 & CAMSCO \\
\cline { 2 - 4 } & Shield de relés 5Vdc & 1 & \\
\cline { 2 - 4 } & Regulador de voltaje & 1 & \\
\cline { 2 - 4 } & PCB & 1 & NEXTION \\
\cline { 2 - 4 } & Pantalla TFT & 1 & ROLELER \\
\cline { 2 - 4 } & Borneras & 50 & \\
\cline { 2 - 4 } & Micro Limit switch & 1 & VARIOS \\
\cline { 2 - 4 } & Sensor Fotoeléctrico & & \\
\cline { 2 - 4 } & Conectores, cable, canaleta, riel DIM, etc. & & \\
\hline
\end{tabular}

\subsection{Circuito neumático}

La Figura 2, muestra el circuito neumático mediante el cual se logra complementar el funcionamiento de los sistemas de distribución, de elevación, de sujeción y de corte.

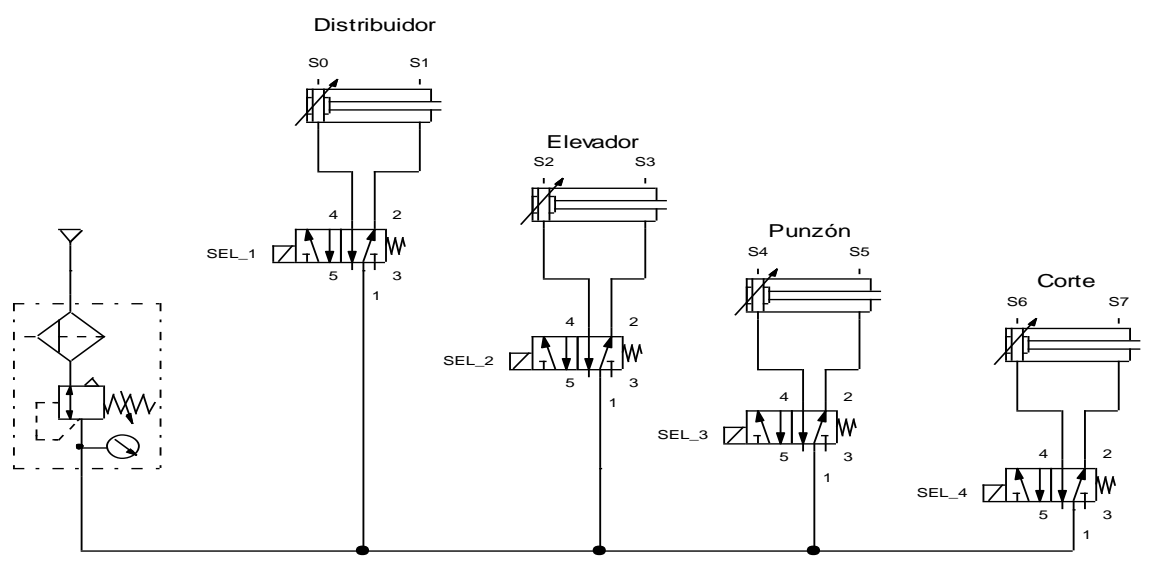

Figura 2. Circuito Neumático. 


\subsection{Tarjeta de control}

El diseño virtual de la tarjeta/placa de control se lo realiza con ARES, todos los elementos fueron dimensionados exactamente para que coincidan con los componentes reales, en la Figura 3, se muestra el diseño final de la placa.

El circuito de potencia está aislado del circuito de control mediante un optoacoplador (MOC3021); este circuito integrado protegerá al microcontrolador de corrientes parásitas que puedan causar daños, la tarjeta de control tiene 6 circuitos de potencia independientes para cada uno de los actuadores.

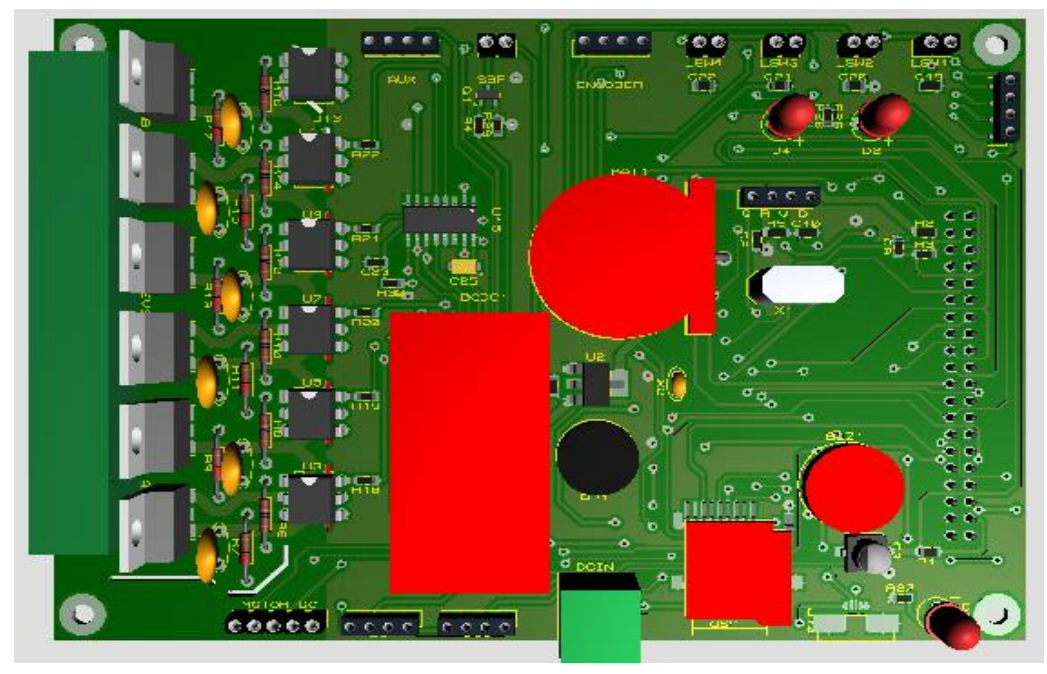

Figura 3. Tarjeta de control.

\subsection{Control del motor sujetador}

Para controlar el cambio de giro del motor DC, se utilizan dos relés como se muestra en la Figura 4. Cuando se encuentra activado el relé RL1, se desactiva RL2 el motor gira en sentido horario y cuando se desactiva RL1 y se activa RL2 el motor gira en sentido antihorario.

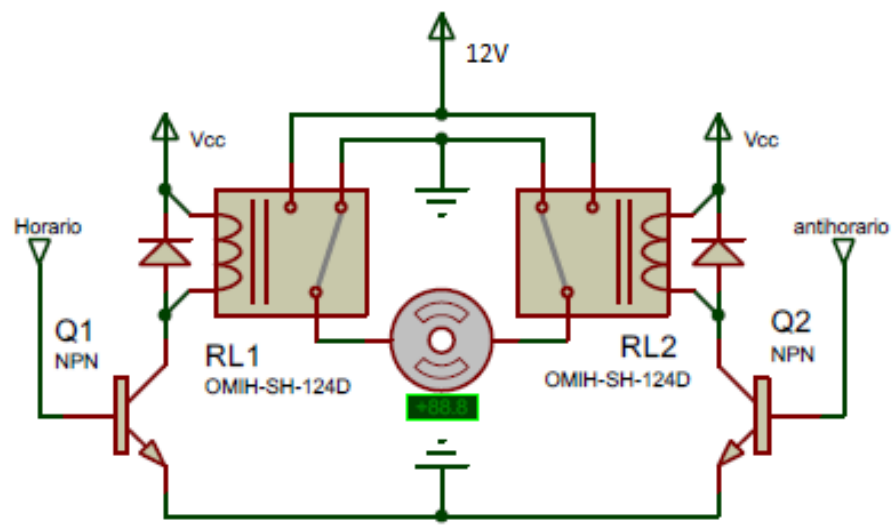

Figura 4. Circuito de control motor sujetador.

\subsection{Lógica de control}

La Figura 5, muestra en forma general el diagrama de flujo del sistema de control implementado para el funcionamiento de la máquina, desde el ingreso de la botella al 
inicio del proceso, pasando por las diferentes etapas de corte, enrollado de los hilos o fibras y la eliminación o descarte del residuo.

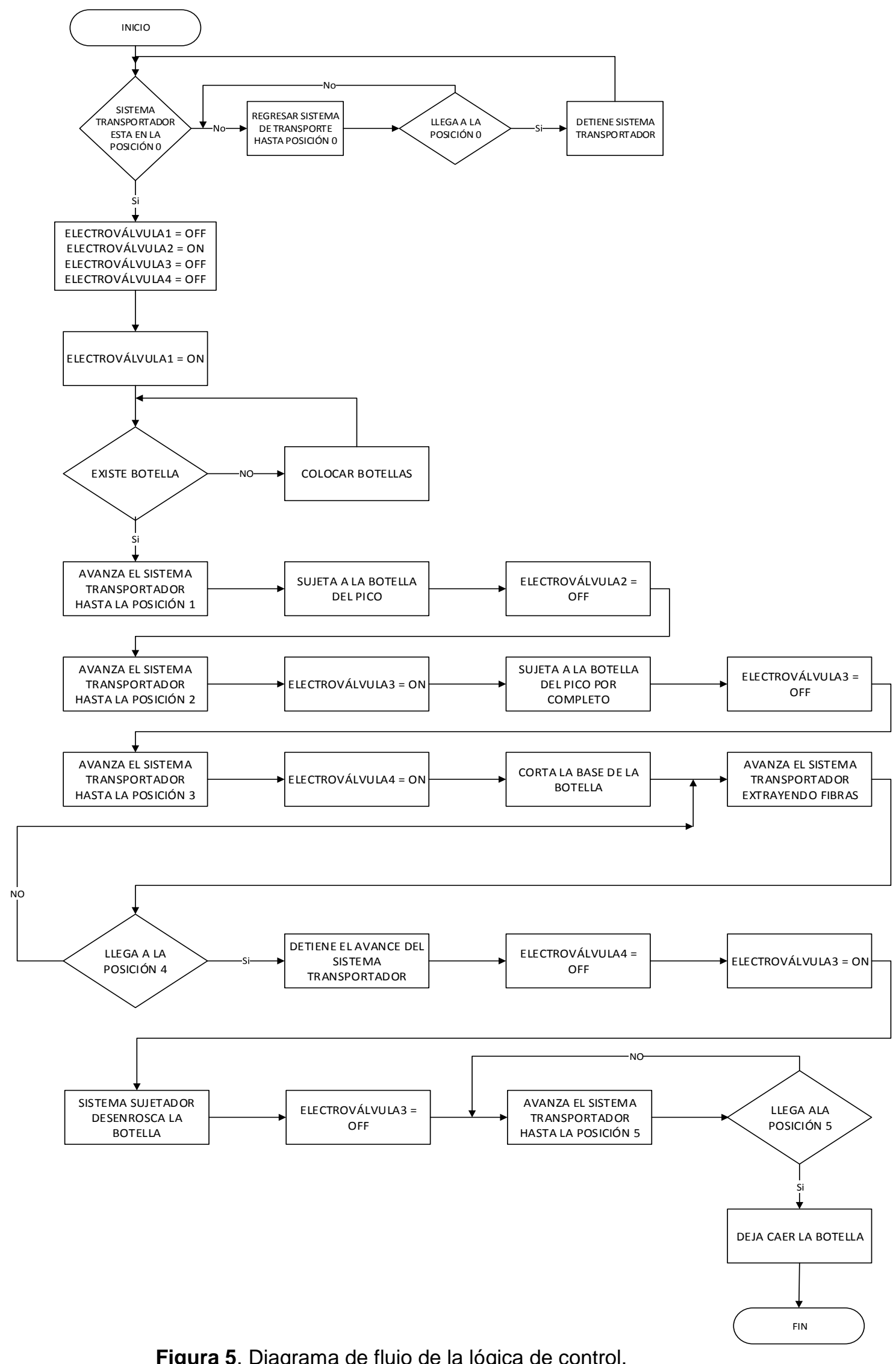




\subsection{Pruebas realizadas}

\subsubsection{Tiempo de Corte}

Las pruebas de corte se realizaron tanto en modo automático como en semiautomático.

En el corte semiautomático, el operario enrolla las fibras obtenidas utilizando el motor que se encuentra instalado en la máquina, mientras que en el corte automático, las fibras obtenidas no son enrolladas por el operario.

El tiempo de corte se tomó desde el arranque de la máquina y se consideraron cinco diferentes valores de frecuencia, estos valores determinan el grosor de la fibra, en la Tabla 6, donde se muestra el tiempo promedio que tarda una botella en ser cortada.

Tabla 6. Tiempo de corte promedio por botella.

\begin{tabular}{|c|c|}
\hline \multicolumn{2}{|c|}{ Tiempo de corte, funcionamiento semiautomático } \\
\hline Frecuencia & Tiempo que se tarda en procesar una botella \\
\hline $3 \mathrm{~Hz}$ & $2 \min 57 \mathrm{~s}$ \\
\hline $4 \mathrm{~Hz}$ & $2 \min 30 \mathrm{~s}$ \\
\hline $5 \mathrm{~Hz}$ & $2 \min 20 \mathrm{~s}$ \\
\hline $6 \mathrm{~Hz}$ & $2 \min 12 \mathrm{~s}$ \\
\hline $7 \mathrm{~Hz}$ & $2 \min 03 \mathrm{~s}$ \\
\hline \multicolumn{2}{|c|}{ Tiempo de corte, funcionamiento automático } \\
\hline Frecuencia & Tiempo que se tarda en procesar una botella \\
\hline $3 \mathrm{~Hz}$ & $2 \min 02 \mathrm{~s}$ \\
\hline $4 \mathrm{~Hz}$ & $1 \min 35 \mathrm{~s}$ \\
\hline $5 \mathrm{~Hz}$ & $1 \min 24 \mathrm{~s}$ \\
\hline $6 \mathrm{~Hz}$ & $1 \min 17 \mathrm{~s}$ \\
\hline $7 \mathrm{~Hz}$ & $1 \min 05 \mathrm{~s}$ \\
\hline
\end{tabular}

\subsubsection{Grosor de la fibra}

Las pruebas realizadas reflejan que el grosor de la fibra está en función de la frecuencia es decir en función de la velocidad del motor. En la Tabla 7, se registran los grosores promedios obtenidos en el corte.

Tabla 7. Grosor de las fibras obtenidas.

\begin{tabular}{|c|c|}
\hline \multicolumn{2}{|c|}{ Grosor de la fibra } \\
\hline Frecuencia & Grosor obtenido \\
\hline $3 \mathrm{~Hz}$ & $1.6 \mathrm{~mm}$ \\
\hline $4 \mathrm{~Hz}$ & $2.8 \mathrm{~mm}$ \\
\hline $5 \mathrm{~Hz}$ & $4.0 \mathrm{~mm}$ \\
\hline $6 \mathrm{~Hz}$ & $5.2 \mathrm{~mm}$ \\
\hline $7 \mathrm{~Hz}$ & $6.7 \mathrm{~mm}$ \\
\hline
\end{tabular}

\subsubsection{Longitud de la fibra}

La longitud promedio de fibra obtenida por botella se encuentra en la Tabla 8, como se puede observar, depende también de la frecuencia, puesto que a menor grosor de fibra, seguro se va a obtener mayor longitud de la misma por botella. 
Tabla 8. Longitud de las fibras obtenidas.

\begin{tabular}{|c|c|}
\hline \multicolumn{2}{|c|}{ Longitud de fibras obtenidas por botella } \\
\hline Frecuencia & Longitud obtenida \\
\hline $3 \mathrm{~Hz}$ & $46.5 \mathrm{~m}$ \\
\hline $4 \mathrm{~Hz}$ & $27.5 \mathrm{~m}$ \\
\hline $5 \mathrm{~Hz}$ & $18.6 \mathrm{~m}$ \\
\hline $6 \mathrm{~Hz}$ & $14.8 \mathrm{~m}$ \\
\hline $7 \mathrm{~Hz}$ & $11.3 \mathrm{~m}$ \\
\hline
\end{tabular}

\subsubsection{Máquina, producto y aplicaciones}

En la Figura 6, se muestran: la máquina terminada, cuatro tipos de fibras o hilos que se pueden obtener en la misma, así como su aplicación inmediata como hilos sujetadores de las ramas en plantaciones de uvilla de la zona de Tabacundo, provincia de Pichincha, Ecuador.

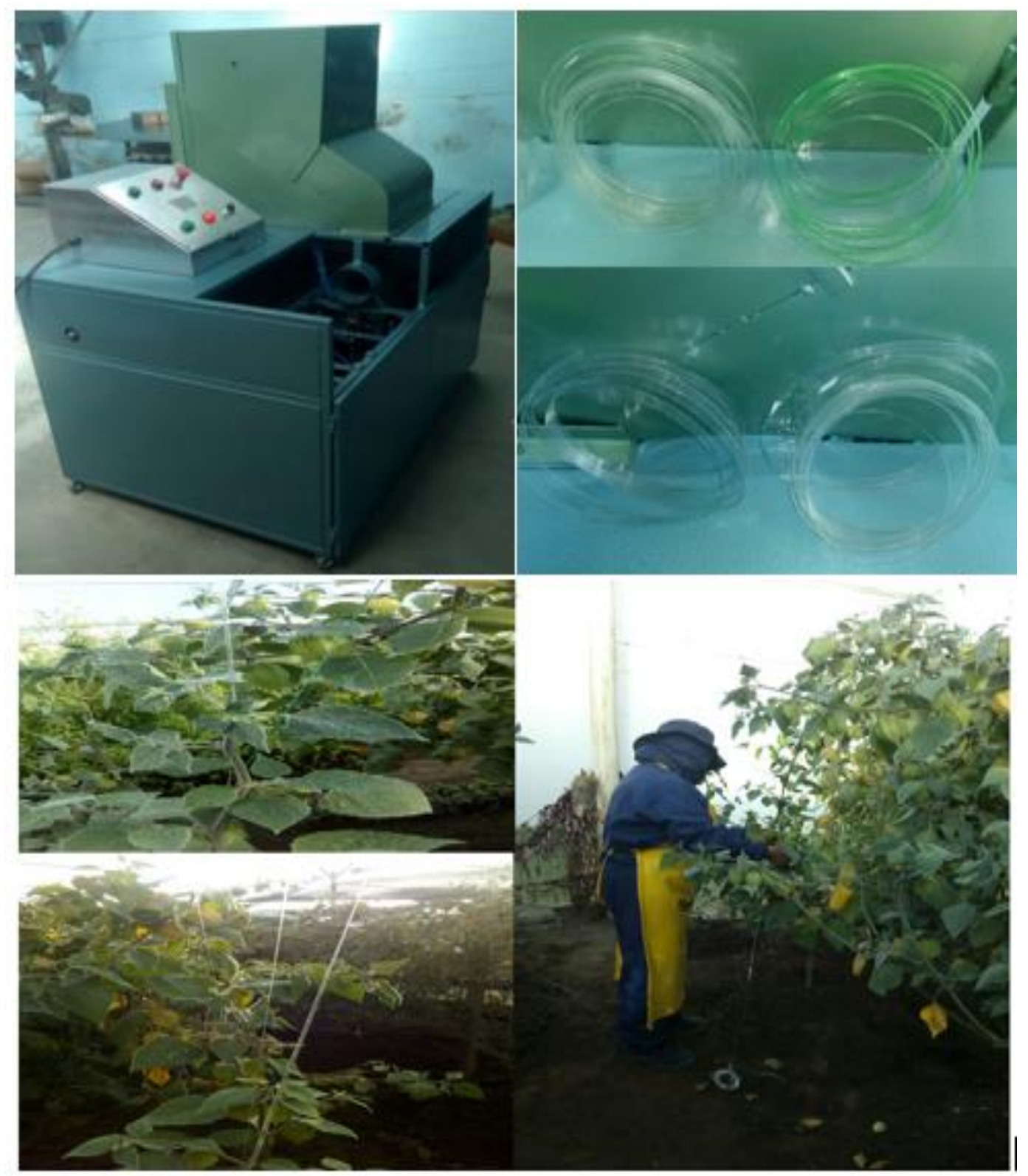

Figura 6. Máquina, producto y aplicaciones. 
De acuerdo con los resultados obtenidos en las diferentes pruebas realizadas, la máquina tiene la posibilidad de trabajar tanto en modo automático como en semiautomático, posibilidades que no se encuentran en otras máquinas con similares propósitos es decir de reciclado por corte rotacional.

En modo automático se pueden procesar 30 botellas de tres litros por hora; se obtiene una longitud de hilo que está en función del grosor de la fibra, grosor que se calibra al variar la velocidad del motor por medio de la modificación de su frecuencia eléctrica.

Una de las principales ventajas de esta máquina automática es la producción de fibras de baja variación en el espesor, lo que es una característica de calidad y de seguridad en el funcionamiento de acuerdo con las aplicaciones o usos que se les de a estas fibras, característica que no se la puede obtener en otras máquinas de este tipo especialmente porque son manuales.

\section{Conclusiones y recomendaciones}

Se logró diseñar y construir una máquina automática (dispositivo mecatrónico) que cumple con los requerimientos de la sociedad actual, con el propósito de reciclar elementos que no son biodegradables y que al no ser correctamente gestionados se convierten en agentes de contaminación ambiental a gran escala, como es el caso de las botellas plásticas.

Tanto el tiempo de proceso de corte, como el grosor o espesor y la longitud de la fibra obtenida de una botella de tres litros depende de la velocidad del tornillo de potencia del sistema de transporte, variable que a su vez depende de la frecuencia eléctrica con la que se le ponga a trabajar al motor que lo acciona.

Por la diversidad de grosores o ancho de fibra que se puede obtener, también existen una diversidad de aplicaciones o usos que se les puede dar a estos productos de manera directa o indirecta, tales como: elementos de sujeción o de soporte, materia prima para la elaboración de cuerdas o sogas, materia prima para la elaboración de escobas, alfombras y recipientes contenedores como fundas, carteras y maletas.

En cuanto al costo de fabricación de la máquina, este puede reducirse al ser producida en serie y en cantidades mayores a un. De esta manera se facilita a personas naturales o jurídicas que con un mínimo de inversión decidan tener una fuente de generación de recursos económicos; al mismo tiempo se contribuye al cuidado del ambiente.

Sobre esta máquina se pueden realizar modificaciones que permitan dotarle de mayor versatilidad para procesar botellas plásticas de diferentes capacidades volumétricas, diferentes diámetros o secciones variables.

\section{Bibliografía}

Bolton, W. (2016). Mecatrónica. México: Alafaomega.

Budynas, R., \& Nisbett, J. (2008). Diseño en Ingeniería Mecánica. México: McGraw Hill Interamericana.

Damián Eneque, O. D. (2016). Implementación de una máquina generadora de hilo ecológico para la disminución de los desechos plásticos y el desarrollo sostenible de las playas del distrito de pimentel. Revista Científica. Ingeniería. Ciencia, Tecnología e Innovación, 30-36.

Flores, E. (2009). ingenieriadesoftware.mex.tl. Obtenido de ingenieriadesoftware.mex.tl: http://ingenieriadesoftware.mex.tl

García Villalba, L. A., Ponce Corral, C., Martínez López, E. J., \& León Ordaz, J. (2016). Diseño y prototipo de una máquina trituradora de PET. Culcyt. Cultura $y$ Tecnología, 63-71. 
Geyer, R., Jambeck, J., \& Lavender Law, K. (2017). Production, use, and fate of all plastics ever made. Science Advances, 1,2.

Gómez González, S. (2010). SolidWorks Simulation. México: Alfaomega.

INEC. (2015). Compendio Estadístico 2015. Quito.

Inteco. (01 de 06 de 2009). jmpovedar.files.wordpress.com. Obtenido de jmpovedar.files.wordpress.com:

https://jmpovedar.files.wordpress.com/2014/03/curso-de-introduccic3b3n-a-laingenieria-del-software.pdf

Nadales Díaz, E. D. (2017). Fortalecimiento de la Cultura del Reciclaje y la Reutilización desde la Axiología Ambiental. Revista Scientific, 133-153.

Pereira de Oliveira, L., \& Castro-Gomes, J. (2011). Physical and mechanical behaviour of recycled PET fibre reinforced mortar. Construction and Building Materials, 17121717.

Serrano, N. (2009). Neumática Práctica. Madrid: Paraninfo.

Shen, L., Worrell, E., \& Patel, M. K. (2010). Open-loop recycling: A LCA case study of PET bottle-to-fibre recycling. Resources, Conservation and Recycling, 34-52.

Siemens. (Mayo de 2015). CatálogoProductosSiemens_Mayo2015.Pdf. Obtenido de CatálogoProductosSiemens_Mayo2015.Pdf:

https://www.industry.siemens.com/home/aan/es/argentina/Documents/CatalogoPr oductosSiemens_MAY2015.pdf

Tojeiro Calaza, G. (2014). Proteus: Simulación de circuitos electrónicos y microcontroladores a través de ejemplos. Marcombo S.A.

Valentín Labarta, J. L. (2012). Introducción a los circuitos neumáticos. Donostiarra. 